\title{
Mucosal sulfhydryl compounds evaluation by in vivo electron spin resonance spectroscopy in mice with experimental colitis
}

\author{
H Togashi, K Oikawa, T Adachi, K Sugahara, J Ito, T Takeda, H Watanabe, K Saito, \\ T Saito, T Fukui, H Takeda, H Ohya, S Kawata
}

Gut 2003;52:1291-1296

\begin{abstract}
Background: Sulfhydryl (SH) compounds are essential in maintaining mucosal integrity in the gastrointestinal tract. A decrease in colonic mucosal SH compounds affects the redox status of the mucosa, resulting in vulnerability to further attacks. Therefore, there is a strong need for in vivo evaluation of $\mathrm{SH}$ compounds in the colonic mucosa.

Aims: The aim of the current study was to establish a method of evaluating levels of SH compounds in the colonic mucosa of live animals before and after induction of colitis.

Methods: Murine experimental colitis was induced by instillation of trinitrobenzene sulphonic acid (TNBS) dissolved in 50\% ethanol into the colon via the anus. For evaluation of mucosal SH compounds in the colon, 3- carbamoyl-2,2,5,5-tetramethylpyrrolidine-1-oxyl (carbamoyl-PROXYL), a stable nitroxide radical, was instilled into the colonic lumen of live mice and the spin clearance rate was measured by L-band electron spin resonance (ESR) spectroscopy. Results: Morphological study showed that mucosal damage was severe one or two days after TNBS instillation. The colonic mucosa started to regenerate at four days, and looked normal at seven days, after induction of colitis. The spin clearance rate of carbamoyl-PROXYL decreased significantly at 0.5 , 1,2 , and 4 days after induction of colitis compared with mice before TNBS instillation. Surprisingly, although the colonic mucosa looked normal seven days after TNBS administration, the spin clearance rate still remained significantly slow. The spin clearance rate returned to normal 14 days after induction of colitis. The change in in vivo spin clearance rate was consistent with the time dependent change in mucosal reduced glutathione, a major component of $\mathrm{SH}$ compounds.

Conclusion: The spin clearance rate obtained by L-band ESR spectroscopy in combination with carbamoyl-PROXYL can give an estimate of the level of colonic mucosal SH compounds in live animals and is useful for evaluating the mucosal defence system against oxidative stress.
\end{abstract}

See end of article for authors' affiliations

Correspondence to: Dr H Togashi, Second Department of Internal Medicine, Yamagata University School of Medicine, 2-2-2

lida-Nishi, Yamagata 990-9585, Japan; htogashi@

med.id.yamagata-u.ac.jp 3 April 2003

eas

nflammatory bowel disease (IBD), including Crohn's disease and ulcerative colitis, is clinically characterised by chronic inflammation in the large and/or small intestine, the symptoms of which include diarrhoea, abdominal pain, weight loss, and nausea. IBDs are complex multifactorial traits involving both environmental and genetic factors. ${ }^{1}$ In patients with IBD, as well as in animals with experimental colitis, decreased mucosal sulfhydryl ( $\mathrm{SH}$ ) compounds, including reduced glutathione (GSH), have been reported. ${ }^{23}$ The comparative scarcity of $\mathrm{SH}$ compounds further increases the vulnerability of the colon towards the deleterious activity of reactive oxygen species (ROS), including lipid peroxidation, oxidative modification of proteins, and DNA alterations. However, evaluation of colonic mucosal SH compounds has been limited to dissected animal samples or biopsied specimens. ${ }^{34}$ In practice, there is a great need for a non-invasive technique suitable for in vivo evaluation of mucosal SH compounds in diseased animal models and humans.

In the current study we investigated mucosal SH compounds in the colon of live mice after treatment with 2,4,6-trinitrobenzene sulphonic acid (TNBS). ${ }^{5}$ We have previously shown that 3-carbamoyl-2,2,5,5-tetramethylpyrrolidine-1-oxyl (carbamoyl-PROXYL), an electron spin resonance (ESR) active compound, could be used as a spin probe for monitoring the availability of glutathione in live mice. ${ }^{56}$ SH compounds, including GSH and cysteine, can reduce carbamoyl-PROXYL to the corresponding hydroxylamine, resulting in the disappearance of the ESR signal of the carbamoyl-PROXYL. When SH compounds in some organs are markedly lowered by harmful insults, the reduction rate of carbamoyl-PROXYL in the organs is significantly delayed. The dynamic change in $\mathrm{SH}$ compounds is estimated by the reduction rate (spin clearance rate) of carbamoyl-PROXYL. For monitoring carbamoyl- PROXY reduction in the targeted organ, we used an ESR apparatus operating at low frequency (700 MHz) which measured the dynamics of free radicals in the live animals. ${ }^{78}$ In the present study, we succeeded in evaluating $\mathrm{SH}$ compounds of injured, and subsequently regenerated, colonic mucosa in TNBS induced colitis in live mice.

\section{MATERIALS AND METHODS}

Induction of colitis

The Animal Studies Ethic Committee of Yamagata University School of Medicine, Japan, approved all of the experiments. C57BL6 mice weighing 20-22 g were fed a standard diet and water. Colitis was induced using a previously described technique. ${ }^{90}$ In fasted C57BL6 mice lightly anaesthetised with ether, a $3.5 \mathrm{~F}$ catheter was inserted into the colon via the anus ( $3 \mathrm{~cm}$ from the anus). TNBS ( $1 \mathrm{mg} /$ mouse; Sigma

\footnotetext{
Abbreviations: carbamoyl-PROXYL, 3-carbamoyl-2,2,5,5-tetramethylpyrrolidine-1-oxyl; ESR, electron spin resonance; GSH, reduced glutathione; IBD inflammatory bowel disease; NEM, $\mathrm{N}$-ethylmaleimide; $\mathrm{SH}$, sulfhydryl; TNBS, trinitrobenzene sulphonic acid; ROS, reactive oxygen species.
} 
A

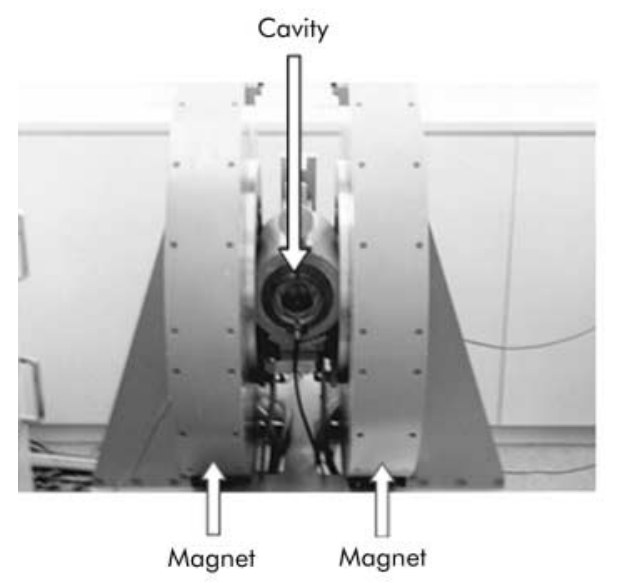

B

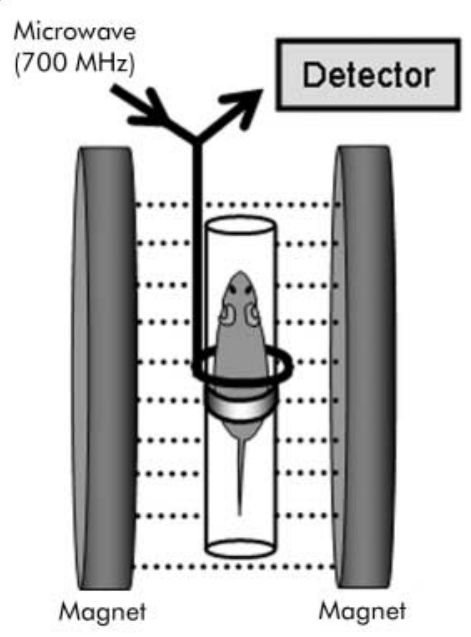

Figure 1 (A) Photograph of the L-band electron spin resonance spectrometer system. (B) Schema of positioning the mice and the electromagnet. C57/BL6 male mice under pentobarbital anaesthesia were placed in the holder in an abdominal position. The proximal part of the hind leg of the mouse was placed at the axial centre of the loop gap resonator. The dotted lines represent the magnetic field gradient.

Chemical Co., St Louis, Missouri, USA) was dissolved in 50\% ethanol $(\mathrm{vol} / \mathrm{vol})$ and instilled $(0.1 \mathrm{ml})$ into the lumen of the colon through the rubber catheter. This procedure required 60 seconds and the treated mice were kept in the Trendelenburg position to facilitate internal retention of the TNBS solution. The mice were returned to their cages. Animals were sacrificed at $1,2,4,7$, and 14 days after TNBS instillation treatment.

\section{Evaluation of colonic damage}

A segment of the colon, $3 \mathrm{~cm}$ long, was excised for macroscopic damage evaluation. After removal, the colon was gently rinsed with saline solution, opened with a longitudinal incision, and pinned out onto a silicon block. The colon was lightly stained with methylene blue and examined under a stereomicroscope. Visible colonic damage was scored on a $0-5$ scale adapted to the murine model by two independent observers. ${ }^{11}$ The following morphological criteria were taken into consideration: score 0, no damage; score 1, localised hyperaemia without ulcers; score 2, linear ulcers with no significant inflammation; score 3, linear ulcer with inflammation at one site; score 4, two or more sites of ulceration and/or inflammation; and score 5, two or more major sites of inflammation and ulceration or one major site of inflammation and ulceration extending more than $1 \mathrm{~cm}$ along the length of the colon.

\section{Colonic GSH measurement}

Colonic tissues were minced in a test tube on ice and homogenised by a Teflon coated Potter-Elvehjem homogeniser with $0.1 \mathrm{M}$ potassium phosphate buffer $(\mathrm{pH} 7.0)$ containing $1 \mathrm{mM}$ EDTA. The homogenates were mixed with $0.6 \mathrm{~N} \mathrm{HClO}_{4}$ containing $1 \mathrm{mM}$ EDTA. The mixtures were centrifuged at $3000 \mathrm{rpm}$ for 10 minutes at $4^{\circ} \mathrm{C}$ and the supernatants were used for GSH measurement. The interaction of GSH with 5, $5^{\prime}$ dithiobis-(2-nitrobenzoic acid) was measured spectrophotometrically at $412 \mathrm{~nm} .{ }^{12}$ Results were expressed as nanomoles of GSH per milligram of protein.

\section{In vivo ESR measurement in live mice}

The low frequency ESR apparatus allows in vivo measurement of free radicals in live animals. ${ }^{71314}$ We used a $700 \mathrm{MHz}$ microwave ESR instrument, constructed in our laboratory, which has already been described in detail. ${ }^{74}$ It consists of a main electromagnet (with air core, water cooled, and a two coil Helmholz design), a pair of field gradient coils, power supplies, a personal computer, and a $700 \mathrm{MHz}$ microwave ESR unit which contained a two gap loop gap resonator and modulation (fig 1A). The magnetic field can be scanned by regulating the current through the field scan coils at a maximum scan rate of $15 \mathrm{mT} / \mathrm{s}$. Linear magnetic field gradients along the $\mathrm{x}, \mathrm{y}$, and $\mathrm{z}$ axes can be produced by the gradient coil (up to $1 \mathrm{mT} / \mathrm{cm}$ in a range from the centre to $20 \mathrm{~mm}$ ).

Each mouse was anaesthetised by intraperitoneal injection of pentobarbital $(50 \mathrm{mg} / \mathrm{kg})$. Carbamoyl-PROXYL solution (2 $\mathrm{mM}, 0.1 \mathrm{ml} / \mathrm{mouse}$ ) was instilled into the colonic lumen via the anus through a rubber cannula. Mice were fixed on a handmade Teflon holder. Immediately after instillation, the ESR spectrum was recorded at the level of the lower abdomen using the ESR spectrometer equipped with a $700 \mathrm{MHz}$ microwave power unit and a loop gap resonator (30 mm diameter) (fig 1B).

The signal decay curve of carbamoyl-PROXYL was obtained by plotting the peak heights of the ESR signals semi logarithmically. Then, the kinetic clearance rate was calculated from the slope of the signal decay curve. ${ }^{615}$ The in vivo spin clearance rate was measured at $0.5,1,2,4,7$, and 14 days after TNBS administration under pentobarbital anaesthesia. The spin clearance rate was measured once for each animal. ${ }^{6}$

\section{Statistical analysis}

All data are expressed as means (SD) unless otherwise indicated. Statistical comparisons were performed using ANOVA, and subsequent comparisons were made using the Mann- Whitney U test. Differences were considered significant at $\mathrm{p}<0.05$.

\section{RESULTS}

The ESR spectrum of carbamoyl-PROXYL has three hyperfine structure lines due to the N-14 nucleus (fig 2A). CarbamoylPROXYL loses the triplet ESR signal by conversion to its hydroxylamine, which produces no ESR signal (fig 2A). A low frequency band ESR (L- band ESR) apparatus allows in vivo measurement of free radicals in live animals. Consequently, the L-band ESR apparatus can monitor the metabolic dynamics of carbamoyl- PROXYL after in vivo administration. Figure 2B shows a typical example of carbamoyl- PROXYL decay after instillation into the murine colon. Immediately after its administration, carbamoyl-PROXYL begins to lose the ESR signal. A semi logarithmic plot of the time course of the ESR signals yielded a signal decay curve for carbamoyl-PROXYL. The curve was almost linear over the first five minutes of 
A

Carbamoyl-PROXYL<smiles>CC1(C)CC(C(N)=O)C(C)(C)N1[O]</smiles>

ESR spectra of carbamoyl-PROXYL

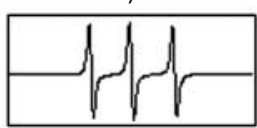

Hydroxylamine

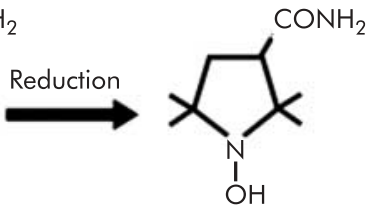

ESR spectra of hydroxylamine
B

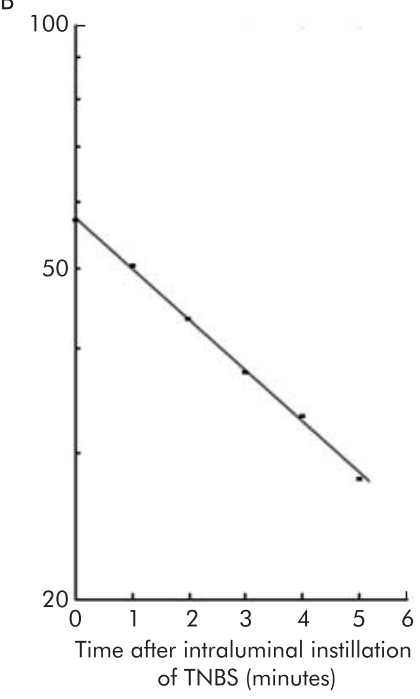

Frequency. $722.669 \mathrm{MHz} \quad$ Power: $20.0 \mathrm{~mW}$

Field: $25.65+5.0 \mathrm{mT}$ Sweep time: $10.0 \mathrm{~s}$

Recelvergain: $32.0 \quad$ Modulation width: $0.1 \mathrm{mT}$

Time constant $0.03 \mathrm{~s}$

Figure 2 (A) Chemical structure of 3-carbamoyl-2,2,5,5-tetramethylpyrrolidine-1-oxyl (carbamoyl-PROXYL) and its corresponding hydroxylamine. The electron spin resonance (ESR) spectrum of carbamoyl-PROXYL has three hyperfine structure lines due to the N-14 nucleus. When carbamoyl-PROXYL is reduced to the corresponding hydroxylamine, this results in the disappearance of the ESR signal. (B) Decrease in ESR signal intensity after intracolonic instillation into a live mouse. The signal decay curve of carbamoyl-PROXYL was obtained by semi logarithmically plotting the peak heights of the ESR signal. The initial spin clearance rate was calculated from the slope of the signal decay.

decay, allowing calculation of initial velocity as the clearance constant (spin clearance rate) (fig 2B).

In vivo ESR measurement of carbamoyl-PROXYL decay after intracolonic instillation was performed using anaesthetised mice. Immediately after instillation of carbamoylPROXYL, the ESR signal in the colon was high enough to be detected by the L-band ESR spectrometer. However, the ESR signal of carbamoyl-PROXYL nearly disappeared eight minutes after instillation (fig 3A). Subsequent instillation of ferricyanide into the colon resulted in the reappearance of the ESR signal by the reoxidation of hydroxylamine ( fig 3A).

Anaesthetised mice were pretreated with $\mathrm{N}$-ethylmaleimide (NEM), an SH blocker, ${ }^{16}{ }^{17}$ intracolonically for 15 minutes using a $3.5 \mathrm{~F}$ catheter and the NEM solution was aspirated as much as possible. Subsequently, carbamoyl-PROXYL was administrated into the colon lumen via the anus. As shown in fig 3B, ESR signal height of carbamoyl-PROXYL did not decrease at eight minutes after intracolonic instillation despite the fact that the carbamoyl- PROXYL signal disappeared at this time in mice without NEM pretreatment.

Intracolonic administration of TNBS resulted in extensive haemorrhage and ulcerative damage to the distal colon, as described previously. Maximal macroscopic damage was present one or two days after TNBS administration (fig 4B, C). However, four days after colitis induction, the mucosal surface of the colon showed healed ulcers and localised erythema, indicative of regeneration of the injured colonic mucosa (fig 4D). Seven days after induction of colitis, the colonic mucosa appeared almost intact both macroscopically and histologically (fig 4E).

Figure 5 shows the results of spin clearance rates measured at various times after induction of colitis. Spin clearance rates significantly slowed at $0.5,1$, and 2 days after induction of colitis by TNBS compared with mice without induction of colitis ( fig 5A). Mucosal damage was the most severe at these times, as indicated by the mucosal damage scores (fig 5A). Four days after induction of colitis, the spin clearance rate was significantly lower. The mucosa started to regenerate but mucosal damage was still present at that time. Although the colonic mucosa regained a normal appearance, both macro-
A
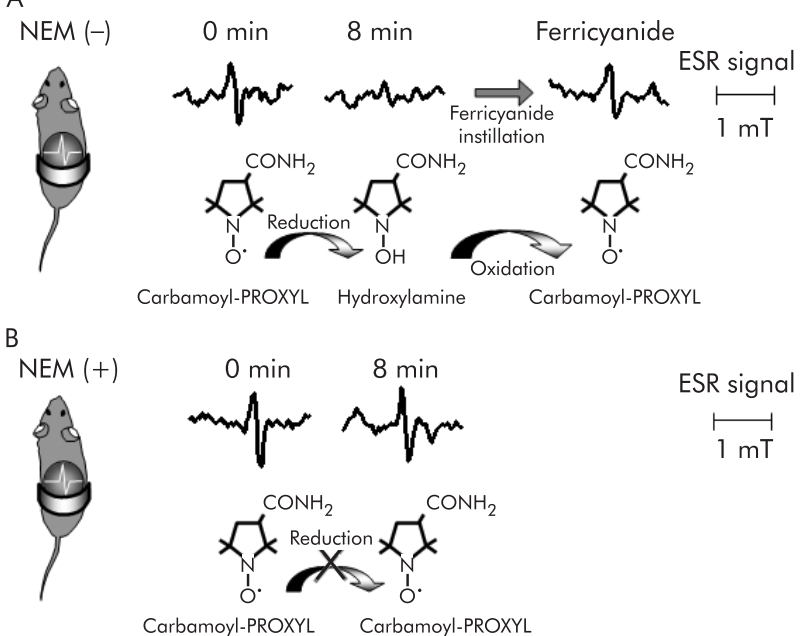

Figure 3 In vivo electron spin resonance (ESR) measurement of 3-carbamoyl-2,2,5,5- tetramethylpyrrolidine-1-oxyl (carbamoylPROXYL) decay by L-band ESR spectroscopy. The peak of the low field component of the triplet signal is demonstrated. (A) The carbamoyl-PROXYL signal peaked initially and decayed rapidly. The ESR signal of carbamoyl- PROXYL cleared eight minutes after instillation. Subsequent instillation of ferricyanide $(3 \mathrm{mM}, 0.05$ $\mathrm{ml} /$ mouse) into the colon caused the ESR signal to reappear. (B) N-ethylmaleimide (NEM) solution $(2 \mathrm{mM}, 0.1 \mathrm{ml} /$ mouse) was instilled into the colonic lumen. Pretreatment with NEM inhibited ESR signal decay in an in vivo experiment.

scopically and microscopically, seven days after colitis induction, the spin clearance rate still remained slower (fig 5A). The spin clearance rate returned to normal 14 days after administration of TNBS (fig 5A).

To examine whether the delay in the spin clearance rate was related to the level of mucosal SH concentration, we determined the time dependent regulation of mucosal GSH levels, one of the major components of SH compounds, in mice treated with TNBS. A progressive fall in mucosal GSH started 

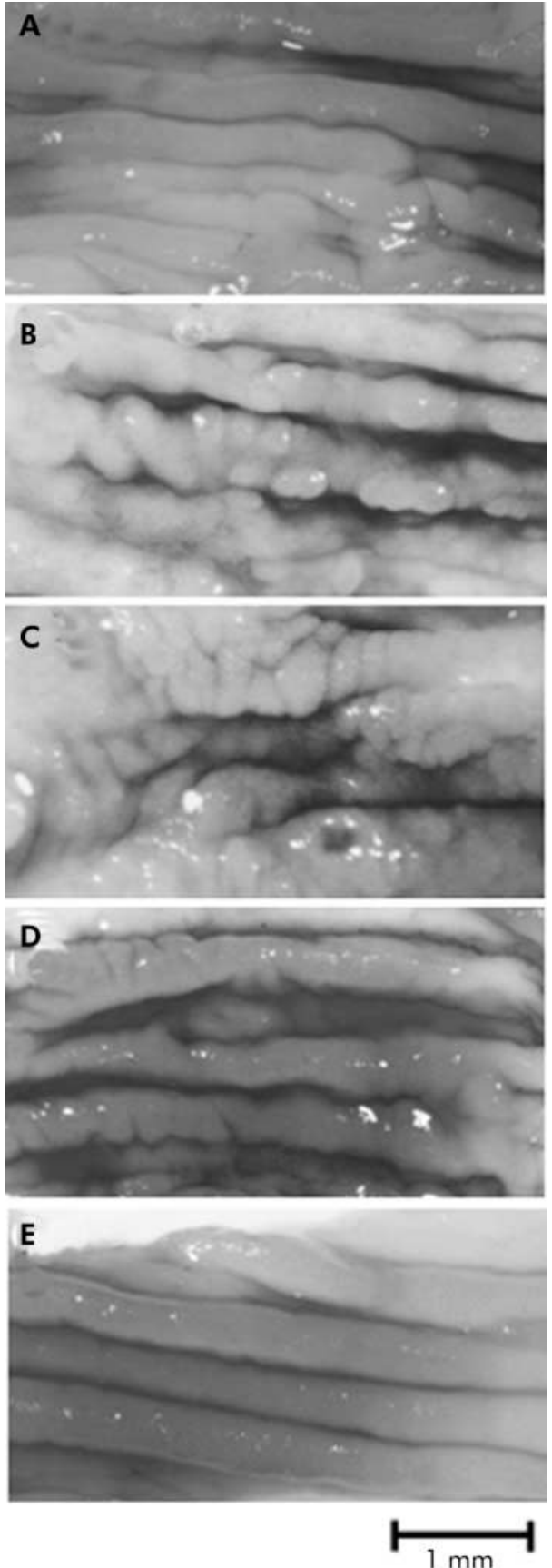

Figure 4 Time course of changes in the colonic mucosa after trinitrobenzene sulphonic acid (TNBS) administration. (A-E) Representative photographs of the colons from mice given $1 \mathrm{mg}$ of TNBS in $0.1 \mathrm{ml}$ of $50 \%$ ethanol and killed at various times afterwards. (A) Normal colonic mucosa. (B, C) Colonic mucosa from a mouse one day (B) and two days (C) after TNBS administration. Acute colonic damage characterised by haemorrhage, ulcers, and bowel wall thickness was present. The ulcers were surrounded by thickened and inflamed mucosa. These colons were given a damage score of 5. (D) Colonic mucosa from a mouse four days after TNBS administration. Mucosal damage was still present, whereas the healing process had started in the colonic mucosa. (E) Colonic mucosa from a mouse seven days after TNBS administration. The colonic mucosa appears normal.

at 12 hours and continued at 1,2,4, and 7 days after treatment with TNBS. As shown in fig 4E, the colonic mucosa regenerated to a normal appearance seven days after treatment with TNBS. The significant decline in mucosal GSH
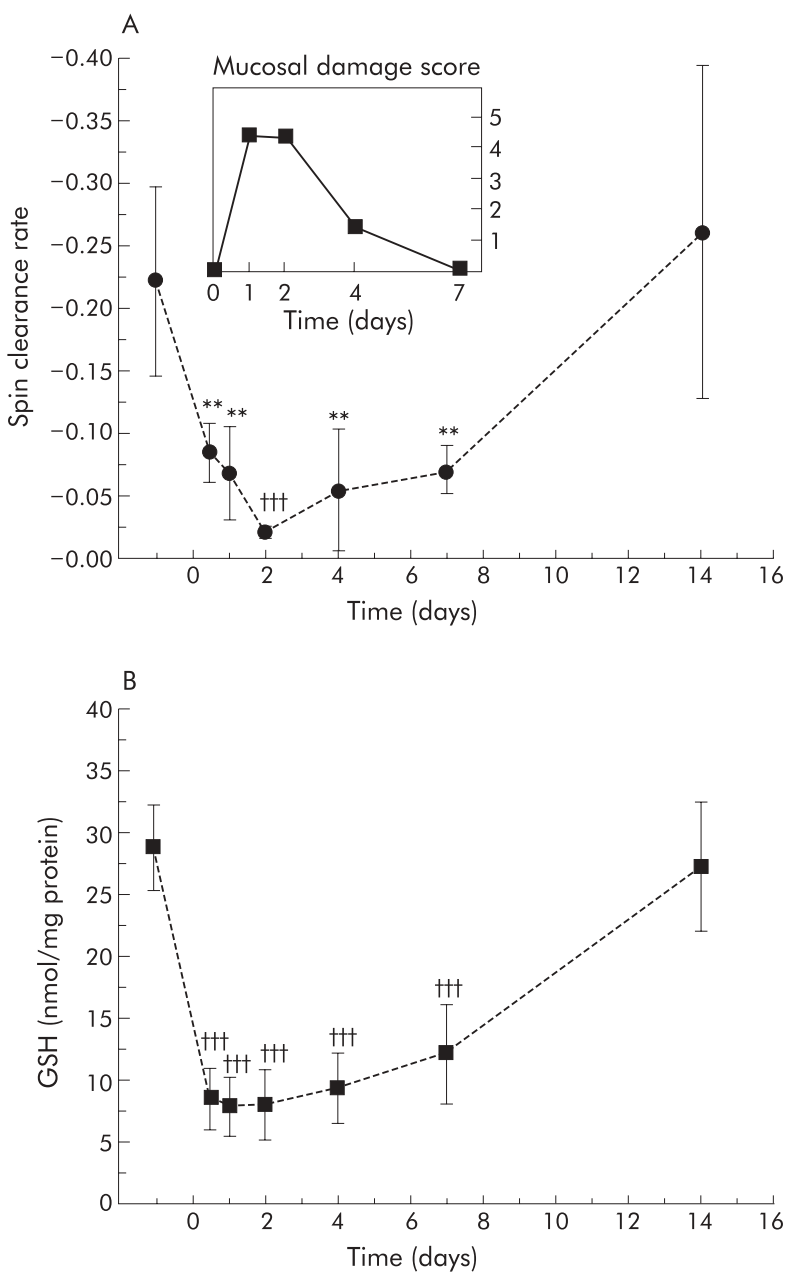

Figure 5 (A) Time course study of the spin clearance rate of 3-carbamoyl-2,2,5,5-tetramethylpyrrolidine-1 -oxyl (carbamoylPROXYL) after trinitrobenzene sulphonic acid (TNBS) instillation into live mice. To help with the understanding of the relationship between mucosal damage and spin clearance rate, the graph of the time course observations of mucosal damage is given. Results are mean (SD) of five mice. There was a significant difference compared with mice without TNBS treatment: $+\dagger p<<0.001,{ }^{* *} p<0.005$. (B) Time course study of mucosal reduced glutathione (GSH) concentrations after TNBS instillation into living mice. There was a significant difference from mice without TNBS treatment: $t+t p<0.001$.

concentration at this point was attributed to the delay in the in vivo spin clearance rate. The mucosal GSH concentration returned to normal 14 days after TNBS administration. The time dependent regulation of mucosal GSH concentration was similar to that of the spin clearance rate.

\section{DISCUSSION}

In this study, we instilled carbamoyl-PROXYL, a stable nitroxide radical, into the colonic lumen of live mice with experimental colitis. Then, the reduction rate (spin clearance rate) of carbamoyl-PROXYL in the colon was examined by L-band ESR spectroscopy. We showed that the spin clearance rate of carbamoyl-PROXYL was closely related to the reduction ability of mucosal SH compounds. The time course study of the spin clearance rate after induction of colitis was similar to the dynamics of mucosal GSH levels, a major component of SH compounds. Moreover, we showed that the spin clearance rate of carbamoyl-PROXYL decreased in the newly regenerated mucosa, which looked morphologically normal seven days after induction of colitis. Therefore, we considered that L-band ESR measurement in combination with carbamoyl-PROXYL 
was an excellent method of estimating the level of mucosal SH compounds in a non-invasive manner, which contributed to maintaining mucosal integrity against various stresses, including oxidative stress.

Nitroxide radicals have six membered ring nitroxides such as TEMPOL, and five membered ring nitroxides such as carbamoyl-PROXYL. The former has a high reactivity against free radicals and provides protection against the harmful effects of free radicals. ${ }^{18}$ In contrast, the latter does not have a high reactivity against free radicals and is used as a contrast medium in ESR imaging. ${ }^{78}$ In theory, nitroxide radicals administered in vivo result in inactive forms by oxidation of nitroxide to oxoammonium cation or reduction to hydroxylamine..$^{19}{ }^{20}$ Our data showed that addition of ferricyanide, which oxidises hydroxylamine to nitroxide, resulted in the reappearance of the ESR signal in in vivo experiments. This indicated that carbamoyl-PROXYL lost its ESR signal through a single electron reduction. Cell membranes are relatively impermeable to ferricyanide. ${ }^{21}$ Therefore, we speculated that most of the carbamoyl-PROXYL might be reduced to hydroxylamine in the colonic lumen by the colonic mucosa Intracolonic administration of NEM, which can react with sulfhydryl (-SH) groups in biomolecules (GSH, cysteine residue in certain proteins, etc), inhibited the reduction of carbamoyl-PROXYL in in vivo experiments. From the results of the present study, SH compounds in the colonic mucosa were key factors in the reduction of carbamoyl-PROXYL.

Hydrogen ion $\left(\mathrm{H}^{+}\right)$is thought to be another factor related to carbamoyl-PROXYL reduction, in addition to $\mathrm{SH}$ groups. $\mathrm{H}^{+}$is secreted into the lumen of the colon by the colonic $\mathrm{H}^{+}-\mathrm{K}^{+}$-ATPase, a member of the P type family of ion transport ATPases. ${ }^{22}{ }^{23}$ Normally, the $\mathrm{H}^{+}$level of the colonic lumen is very high and the luminal environment is kept very reductive. $\mathrm{H}^{+}$in the colonic lumen may affect the in vivo spin clearance rate of carbamoyl-PROXYL. In this study, we did not examine the relation between $\mathrm{H}^{+}$in the colonic lumen and the spin clearance rate of carbamoyl-PROXYL. Further study will be required to clarify this aspect.

Rachmilewitz et al reported that iodoacetamide, an $\mathrm{SH}$ blocker, induced significant mucosal injury after intracolonic administration to rats by increasing the susceptibility to ROS. ${ }^{17}{ }^{24}$ They emphasised the pathophysiological importance of SH compounds in maintaining mucosal integrity. ${ }^{1722}$ The comparative scarcity of SH compounds causes loss of mucosal integrity and further amplifies the vulnerability of the colon towards the deleterious activity of ROS. In patients with IBD, decreased mucosal SH compounds including GSH have been reported. $^{24}$ Therefore, in vivo evaluation of mucosal SH compounds is clinically relevant. Measurement of the spin clearance rate of carbamoyl- PROXYL provides a convenient method of evaluating the level of colonic mucosal $\mathrm{SH}$ compounds, which cannot be achieved by endoscopic examination.

In the colitis model induced by TNBS, the spin clearance rate of carbamoyl-PROXYL measured by L-band ESR spectroscopy was significantly delayed in the presence of obvious mucosal injury. Surprisingly, even if the damaged colonic mucosa appeared apparently normal through a regeneration process, the spin clearance rate was lower at this point, indicating incomplete recovery of $\mathrm{SH}$ compounds in the newly regenerated colonic mucosa. Furthermore, we were able to show that the concentration of mucosal GSH, a major component of SH compounds, decreased at that point. Loguercio et al examined the antioxidant defence in the TNBS induced colitis model and showed a dramatic decrease in GSH unrelated to the destruction of cells during the healing process in TNBS induced colitis. ${ }^{3}$ The report of Loguercio et al and our present data indicate that there is a time lag between morphological recovery and recovery of SH compounds. Here, we showed that the in vivo spin clearance rate mainly reflected the concentration of colonic mucosal SH compounds. The decreased level of mucosal SH compounds may induce susceptibility to the appearance of further acute damage-that is, occurrence of oxidative stress.

As IBDs are characterised by a series of acute attacks superimposed on chronic disease, evaluation of the integrity of the colonic mucosa is important in predicting relapse in IBDs. At present, colonoscopy is the best method for evaluating the degree of mucosal injury in humans. However, evaluation of the regenerated mucosa by colonoscopy is not always satisfactory because it is well known that biopsy of what appears to be intact mucosa may reveal characteristic histological abnormalities. ${ }^{25}$ The present study suggests that the noninvasive in vivo analytical method by L-band ESR spectroscopy provides the potential for clinical applications for evaluation of human colonic mucosa by a method different from colonoscopy. For the clinical application of L-band ESR spectroscopy, we are developing in vivo ESR spectroscopy equipment with a surface coil-type resonator, which can be applicable to endoscopy.

In conclusion, we have described a new method for in vivo evaluation of SH compounds in TBNS induced colitis mucosa. Because L-band ESR spectroscopy, in combination with carbamoyl-PROXYL, can evaluate SH compounds in living animals in a non-invasive manner, it has the potential for clinical applications for the evaluation of human colonic mucosal integrity.

\section{ACKNOWLEDGEMENTS}

The authors thank the reviewers for their helpful comments and suggestions.

Authors' affiliations

H Togashi, T Adachi, K Sugahara, J Ito, T Takeda, H Watanabe, K Saito, T Saito, T Fukui, H Takeda, S Kawata, Second Department of Internal Medicine, Yamagata University School of Medicine, 2-2-2 lida-Nishi, Yamagata 990-9585, Japan

K Oikawa, Yamagata Research Institute of Technology, Yamagata 990-2473, Japan

H Ohya, Institute for Life Support Technology, Yamagata Public Corporation for Development of Industry, Yamagata 990-2473, Japan

\section{REFERENCES}

1 Sartor RB. Pathogenesis and immune mechanisms of chronic inflammatory bowel diseases. Am J Gastroenterol 1997:92:5-11S.

2 Sido B, Hack V, Hochlehnert A, et al. Impairment of intestinal glutathione synthesis in patients with inflammatory bowel disease. Gut 1998;42:485-92.

3 Loguercio C, D'Argenio G, Delle Cave M, et al. Direct evidence of oxidative damage in acute and chronic phases of experimental colitis in rats. Dig Dis Sci 1996:41:1204-11

4 McKenzie SJ, Baker MS, Buffinton GD et al. Evidence of oxidant-induced injury to epithelial cells during inflammatory bowel disease. J Clin Invest 1996;98:136-41.

5 Togashi H, Shinzawa H, Matsuo T, et al. Analysis of hepatic oxidative stress status by electron spin resonance spectroscopy and imaging. Free Radic Biol Med 2000;28:846-53

6 Togashi $\mathbf{H}$, Matsuo T, Shinzawa $\mathrm{H}$, et al. In vivo imaging of increased oxidative stress in the liver by electron spin resonance-computed tomography. Res Commun Mol Pathol Pharmacol 2000;107:197-217.

7 Ishida S, Matsumoto S, Yokoyama H, et al. An ESR-CT imaging of the head of a living rat receiving an administration of a nitroxide radical. Magn Reson Imaging 1992;10:109-14.

8 Togashi $\mathrm{H}$ Shinzawa $\mathrm{H}$, Ogata $\mathrm{T}$, et al Spatiotemporal measurement of free radical elimination in the abdomen using an in vivo ESR-CT imaging system. Free Radic Biol Med 1998;25:1-8.

9 Morris GP, Beck PL, Herridge MS, et al. Hapten-induced model of chronic inflammation and ulceration in the rat colon. Gastroenterology 1989;96:795-803

10 Neurath MF, Fuss I, Kelsall BL, et al. Antibodies to interleukin 12 abrogate established experimental colitis in mice. J Exp Med $1995 \cdot 182 \cdot 1281-90$.

11 Zingarelli B, Szabo C, Salzman AL. Reduced oxidative and nitrosative damage in murine experimental colitis in the absence of inducible nitric oxide synthase. Gut 1999;45:199-209.

12 Moron MS, Depierre JW, Mannerick B. Levels of glutathione, glutathione reductase and glutathione-S-transferase activities in rat liver and lung. Biochim Biophys Acta 1979:582:67-78.

13 Oikawa K, Ogata T, Togashi H, et al. A 3D- and 4D-ESR imaging system for small animals. Appl Radiat Isot 1996;47: 1605-9. 
14 Zweier JL, Kuppusamy P. Electron paramagnetic resonance measurements of free radicals in the intact beating heart: a technique for detection and characterization of free radicals in whole biological tissues. Proc Natl Acad Sci U S A 1988;85:5703-7.

15 Sano T, Umeda F, Hashimoto T, et al. Oxidative stress measurement by in vivo electron spin resonance spectroscopy in rats with streptozotocin-induced diabetes. Diabetologia 1998;41:1355-60.

16 Yamaguchi T, Murata Y, Kobayashi J, et al. Effects of chemical modification of membrane thiol groups on hemolysis of human erythrocytes under hydrostatic pressure. Biochim Biophys Acta 1994; 1195:205-10.

17 Rachmilewitz D, Karmeli F, Okon E. Sulfhydryl blocker-induced rat colonic inflammation is ameliorated by inhibition of nitric oxide synthase. Gastroenterology 1995;109:98-106.

18 Karmeli F, Eliakim R, Okon E, et al. A stable nitroxide radical effectively decreases mucosal damage in experimental colitis. Gut 1995; 37:386-93

19 Chen K, Glockner JF, Morse PD $2^{\text {nd }}$, et al. Effects of oxygen on the metabolism of nitroxide spin labels in cells. Biochemistry 1989:28:2496-501.
20 Krishna MC, Grahame DA, Samuni A, et al. Oxoammonium cation intermediate in the nitroxide-catalyzed dismutation of superoxide. Proc Natl Acad Sci U S A 1992;89:5537-41.

21 Kaplan J, Canonico PG, Caspary WJ. Electron spin resonance studies of spin-labeled mammalian cells by detection of surface-membrane signals. Proc Natl Acad Sci U S A 1973;70:66-70.

22 Suzuki Y, Kaneko K. Acid secretion in isolated guinea pig colon. Am J Physiol 1987;253:G155-64.

23 Crowson MS, Shull GE. Isolation and characterization of a cDNA encoding the putative distal colon $\mathrm{H}+, \mathrm{K}(+)$-ATPase. Similarity of deduced amino acid sequence to gastric $\mathrm{H}+, \mathrm{K}(+)$-ATPase and $\mathrm{Na}+\mathrm{K}(+)$-ATPase and mRNA expression in distal colon, kidney, and uterus. J Biol Chem 1992;267: 13740-48

24 Rachmilewitz D, Okon E, Karmeli F. Sulphydryl blocker induced small intestinal inflammation in rats: a new model mimicking Crohn's disease. Gut 1997:41:358-65.

25 Floren $\mathrm{CH}$, Benoni C, Willen R. Histologic and colonoscopic assessment of disease extension in ulcerative colitis. Scand J Gastroenterol 1987:22:459-62.

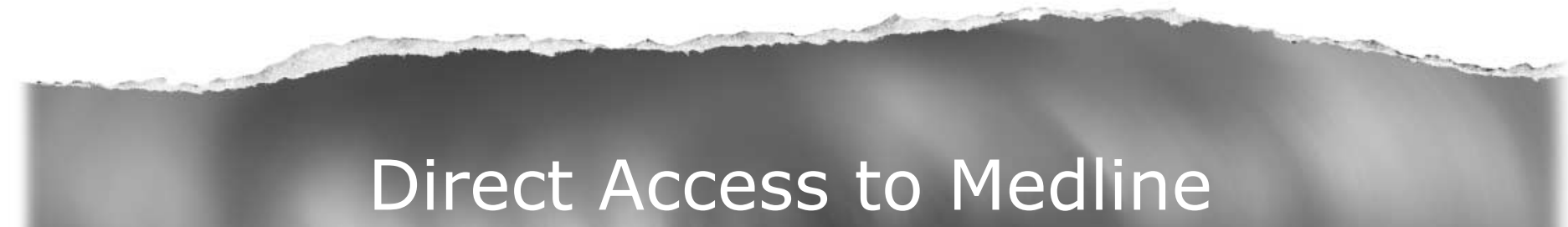

Medline

Link to Medline from the homepage and get straight into the National Library of Medicine's premier bibliographic database. Medline allows you to search across 9 million records of bibliographic citations and author abstracts from approximately 3,900 current biomedical journals.

\section{www.gutjnl.com}

